The average dynamic lift on the bed particles was measured by a similar method to that used in the studies with the hemispheres; that is, the difference of pressures in tap holes of the channel wall above the bed and in perforated tubes under the bed was measured by means of micro-point-gauges. The average lift pressure was found to be again

$$
\Delta p=0.178 \rho\left(u^{2} / 2\right),
$$

if the velocity $u$ again was measured 0.35 -grain diameters from the theoretical wall and the sieve size of that grain size was introduced as the diameter of which 35 percent (by weight) of the mixture was finer. This diameter is considerably different from that describing the roughness of the mixture, but equals that size which the senior author previously found to describe the mixture in an equation for the rate of bed-load load transportation. ${ }^{6}$ Since the lift has been described

${ }^{6}$ H. A. Einstein, Trans. A.S.C.E. 107, 571 (1942). as the major moving force of bed-load, ${ }^{2}$ the agreement of the representative grain diameters for lift and transport of mixtures seems to be significant.

The measurement of the fluctuations of this lift force was not attempted, as the choice of a particle for measurement would have been arbitrary and for that reason hardly sufficiently representative to prove anything.

This paper represents a resume of the results which the junior author found in an experimental study under the general guidance of the senior author and which is described in detail in a Ph.D. thesis. ${ }^{7}$ A copy of this thesis, which includes the description of the apparatus, including many photographs, tables, and graphs, all calibrations and measuring results may be made available on a loan basis to all who are especially interested.

${ }^{7}$ E. A. El Samni, "Hydrodynamic forces acting on the surface particles of a stream bed" (Ph.D. thesis, University of California, Dept. of Eng., Berkeley, 1949).

\title{
On the Transfer Rate of the Rollin-Simon Film
}

\author{
W. J. DE HAas and G. J. VAN DEN BERG \\ Kamerlingh Onnes Laboratory, Leiden University, Holland
}

$I^{\mathrm{N}}$ 1922 Kamerlingh Onnes attempted to reach the lowest possible temperature in the following manner: He surrounded a Dewar flask completely with liquid helium in such a way that heat could not penetrate by radiation nor by conduction. Inside this Dewar flask he placed still another smaller Dewar flask filled partly with liquid helium and he hoped through the lowering of the vapor pressure with molecular pumps of great speed to reach an extremely low temperature.

In this experiment there appeared a most remarkable phenomenon: With amazing rapidity liquid helium appeared outside the inner flask until the two levels of the helium in the inner and outer flask were equal. By varying this experiment he found that helium placed in the outer flask was also transferred to the inner flask. This transfer always continued until the levels in both flasks were at the same height.

The origin of this remarkable Kamerlingh Onnes effect was given by Simon and Rollin. They assumed that the wall of the flask was covered by an extremely thin film of helium and in this way the helium was "syphoned" from one place to the other. This RollinSimon film has been investigated in Oxford, Cambridge, Bristol and Russia.

Daunt and Mendelssohn carried out extensive experiments on the transport phenomena in the He II film; meanwhile Atkins in Cambridge got quite different results. The principal results which Daunt and Mendelssohn obtained were:

1. The transport velocity is a constant quantity independent of the difference in levels except for the case in which the He II in the tube is less than $1 \mathrm{~cm}$ from the top.

2. The transport velocity increases with falling temperature from the $\lambda$-point to $1.5^{\circ} \mathrm{K}$ and remains approximately constant below this temperature.

3. The transport velocity is independent of the material over which the film creeps.

The results of Atkins, however, are quite different. $\mathrm{He}$ finds the maximum transport velocity about 13 times greater than Daunt and Mendelssohn, and moreover this velocity is not constant but depends on the distance of the highest level to the top of the tube. As Atkins did not give many details of his apparatus it is difficult to reach definite conclusions concerning his results.

\section{INVESTIGATIONS AT LEIDEN}

Since the film has a very large surface area it is assumed that its thickness will be sensitive to incident radiation.

In order to avoid the effects of radiation one must reject all direct methods of visual observations. The principle which we therefore adopted as a criterion for measurement was the decrease of weight with time of a tube filled with He II from which the film creeps. 


\section{DESCRIPTION OF THE APPARATUS}

A heavy walled copper tube $C$ (Fig. 1) is submerged in liquid helium which completely fills a Dewar flask. Helium is condensed in this copper tube to an amount sufficiently great so that the two tubes $(G)$ can be completely submersed and thereafter withdrawn. A thin glass rod connects the tubes $(G)$ with the spring $S$. The helium creeps out of the tubes and drips off their bottoms. The resulting loss of weight is measured by the observations with a cathetometer of the point $P$. The spring has been calibrated. The condensed helium entered through tube $D$. The tubes $A$ and $B$ are very narrow and made of german silver. Before the experiments began the tubes $G-G$ were turned through $90^{\circ}$ with respect to the plane of the tubes $A-B$. The liquid helium II in the tubes $G-G$ thereby is quite protected from all internal radiation. The innermost Dewar flask, filled with liquid helium is surrounded by a flask filled with liquid hydrogen and finally still another with liquid nitrogen. All vessels are silvered.

\section{RESULTS}

The determined transfer rate departs from that found by Daunt and Mendelssohn and resembles more that of Atkins.

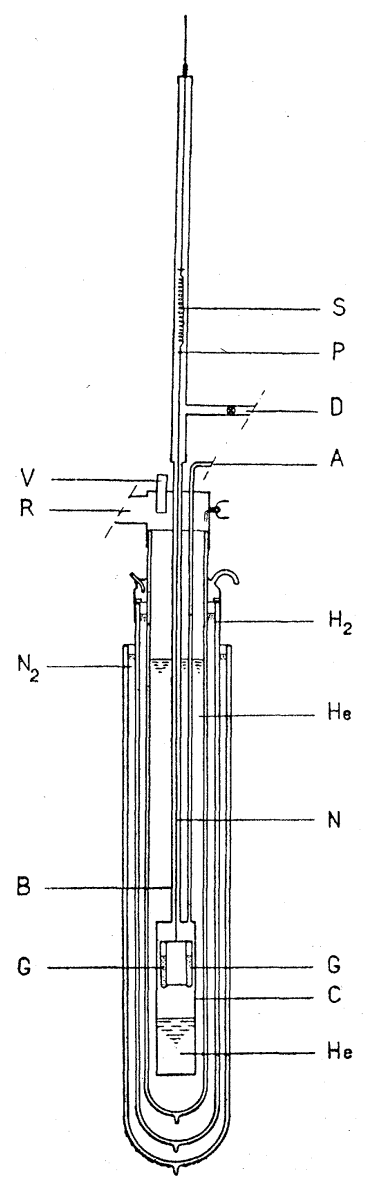

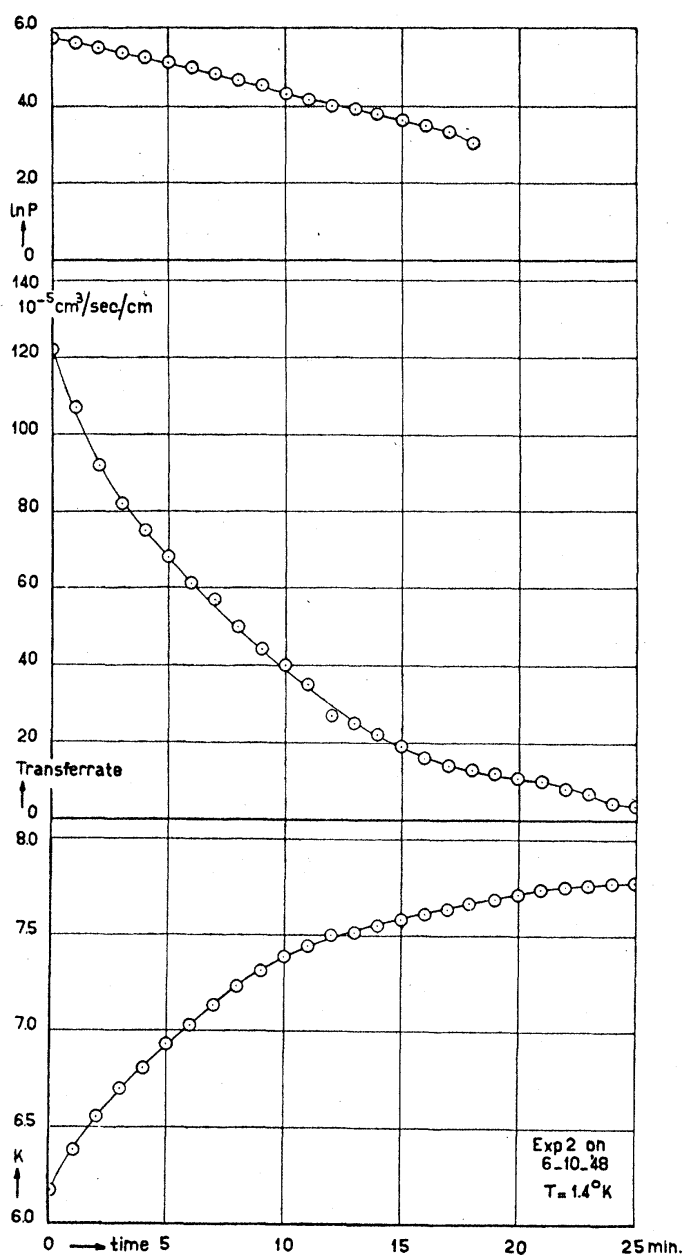

- FIg. 2. Rate of transfer of helium out of container by means of Rollins film.

The transport velocity was found to have an initial value of 130 to $140 \times 10^{-5} \mathrm{~cm}^{3} / \mathrm{sec}$. $/ \mathrm{cm}$ (depending on the precise instant of the first observations) at a temperature of $1.4^{\circ} \mathrm{K}$, while Daunt and Mendelssohn reported the value $7.5 \times 10^{-5} \mathrm{~cm}^{3} / \mathrm{sec} . / \mathrm{cm}$ at the same temperature.

The transfer rate is not constant but tends to zero as the level sinks. The much greater transfer velocity suggests that in our case the film is much thicker than in the case of Daunt and Mendelssohn. It seems plausible that the small amount of radiation still present in the experiments of Daunt and Mendelssohn evaporates the film, and that for this reason the character of the phenomenon changes. In the case of the very thin film the velocity will be determined by the mechanism of the solid-liquid double layer. In the case of the space quite free from radiation with consequent greater thickness of film the bulk of the film will be bound by very small forces and in this case the phenomenon, contrary to the thin film, will show the character of syphoning. If this reasoning is correct then for 
TABLE I. Transfer of He II. (Experiment 2 on 6-10-1948.)

\begin{tabular}{|c|c|c|c|c|c|}
\hline \multirow{3}{*}{$\begin{array}{l}\text { Time } \\
\text { min. }\end{array}$} & \multirow{2}{*}{\multicolumn{2}{|c|}{ Cathetometer readings }} & \multirow{3}{*}{$\begin{array}{c}\text { Transfer rate } \\
10^{-5} \mathrm{~cm}^{3} / \\
\text { sec. } / \mathrm{cm}\end{array}$} & \multirow{3}{*}{$\begin{array}{c}\text { Capacity } P \\
\text { of the vessels } \\
G-G \\
\text { m.g. }\end{array}$} & \multirow[t]{3}{*}{$\ln P$} \\
\hline & & & & & \\
\hline & obs. & calc. & & & \\
\hline 0 & 6.17 & 6.17 & 122 & 320 & 5.77 \\
\hline 1 & 6.38 & 6.39 & 107 & 278 & 5.63 \\
\hline 2 & 6.55 & 6.56 & 92 & 244 & 5.50 \\
\hline 3 & 6.70 & 6.72 & 82 & 214 & 5.37 \\
\hline 4 & 6.81 & 6.85 & 75 & 193 & 5.26 \\
\hline 5 & 6.93 & 6.97 & 68 & 168 & 5.12 \\
\hline 6 & 7.03 & 7.07 & 61 & 149 & 5.00 \\
\hline 7 & 7.14 & 7.16 & 57 & 127 & 4.84 \\
\hline 8 & 7.27 & 7.24 & 50 & 108 & 4.68 \\
\hline 9 & 7.32 & 7.31 & 44 & 91 & 4.51 \\
\hline 10 & 7.39 & 7.37 & 40 & 76 & 4.33 \\
\hline 11 & 7.45 & 7.42 & 35 & 65 & 4.17 \\
\hline 12 & 7.50 & 7.47 & 27 & 54 & 3.99 \\
\hline 13 & 7.52 & 7.51 & 25 & 51 & 3.93 \\
\hline 14 & 7.55 & 7.54 & 22 & 44 & 3.78 \\
\hline 15 & 7.58 & 7.57 & 19 & 38 & 3.64 \\
\hline 16 & 7.61 & 7.60 & 16 & 32 & 3.47 \\
\hline 17 & 7.64 & 7.62 & 14 & 28 & 3.33 \\
\hline 18 & 7.67 & 7.64 & 13 & 21 & 3.04 \\
\hline
\end{tabular}

the weight of the liquid in the tubes $G-G$ denoted by $P$, we may write for the time interval $d t$

$$
d P=-\alpha P d t
$$

or putting the initial value for $P, P_{b}$

$$
\ln \left(P / P_{b}\right)=-\alpha t \text {. }
$$

Hence we get in first approximation for the emptying of the vessels $G-G$ a logarithmic curve (Fig. 2).

Rewriting Eq. (1) in terms of the direct observations of the cathetometer, we calculated the different cathetometer values $K$ as ordinates in Fig. 2.

In Table I the observed and calculated values of $K$ against the time in minutes are given.

The agreement between observed and calculated values is quite satisfactory and as a rule within $\frac{1}{2}$ percent.

The bottom of the vessels $G-G$ are hemispherical. When the level of the liquid enters those hemispherical parts the logarithmic law does not hold.

We must give our attention also to the mechanocaloric effect. As is well known, the temperature of the film $(A)$ (Fig. 3) creeping out of the liquid is lower than the mean value of the temperature, while at the same time at the point $(B)$ of the liquid where the film leaves, the temperature is higher than the mean value. We may assume that the temperature of the film is quickly raised by condensation of helium vapor. Of course we are not certain on this point. To verify the influence of the mechano-caloric effect we should determine the temperature of the liquid in the vessels $G-G$.

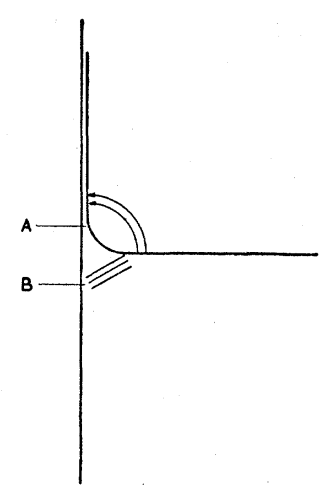

Fig. 3. Boundary of liquid body and film.

We omitted a thermometer in the vessels $G$ - $G$ because of the rather difficult technical complications. However, we do not think this a serious source of errors.

Daunt and Mendelssohn found that no change in the transfer velocity resulted when the tube filled with liquid helium was surrounded by liquid helium II.

Summary: The mechanism of transfer of the RollinSimon film depends greatly upon the presence of a small amount of radiation.

1. In the absence of radiation we get a much greater transfer rate. We determined in the beginning of each experiment values which are as much as seventeen times greater than Daunt and Mendelssohn.

Our investigations agree in this respect more with those of Atkins.

2. The law of transfer is to a first approximation a logarithmic law (syphon action).

Hence the creeping of the film is not constant but decreases steadily from an initial maximum to zero when the vessel is nearly emptied.

The more detailed and extensive experiments will be published in Physica. In this preliminary note only one example is given.

\section{BIBLIOGRAPHY}

H. Kamerlingh Onnes, Commun. Leiden, No. 159; Trans. Faraday Soc. 18, No. 53 (1922).

Oxford:

B. V. Rollin, Act. 7 Int. Cong. Refrig. 1, 187 (1936).

B. V. Rollin and F. Simon, Physica, 's Grav. 6, 219 (1939).

J. G. Daunt and K. Mendelssohn, Proc. Roy. Soc. A170, 423 and 439 (1939); Nature 143, 719 (1939); Phys. Rev. 69, 126 (1946); Nature 157, 839 (1946).

K. Mendelssohn, Nature 160, 385 (1947); Oxford Conf., 1947. J. B. Brown and K. Mendelssohn, Nature 160, 670 (1947). Cambridge:

K. R. Atkins, Nature 161, 925 (1948).

Russia (U.S.S.R.) :

P. Kapitza, Nature 141, 74 (1938)

A. K. Kikoin and B. G. Lasarew, Nature 141, 912 (1938) and 142,289 (1939)

J. Frenkel, J. Phys. U.S.S.R. 2, 365 (1940); J. exp. theor. Phys. (Russ.) 10, 650 (1940).

A. K. Kikoin and B. G. Lasarew, J. Phys. U.S.S.R. 4, 379 (1941).

P. G. Strelkov, J. Phys. U.S.S.R. 4, 379 (1941). 